\title{
Active QoS Flow Maintenance in Controlled Mobile Networks
}

\author{
John D. Sweeney, Roderic Grupen, and Prashant Shenoy \\ Laboratory for Perceptual Robotics \\ Department of Computer Science \\ University of Massachusetts Amherst \\ \{sweeney,grupen, shenoy\}@cs.umass.edu
}

\begin{abstract}
Applications involving teams of mobile robots will require robots within the system to form connections to other members with certain quality of service $(\mathrm{QoS})$ requirements. We present a distributed control architecture that allows robots participating in routing a QoS flow to maintain the required level of service while addressing secondary objectives. A distributed control system preserves global properties using "best-effort", error-suppressing controllers. We outline a routing protocol that dynamically reconfigures a flow by recruiting neighboring robots if it believes a routing fault may occur. We evaluate the control architecture and dynamic configuration protocol in simulation, using the ns-2 network simulator and Player/Stage robot simulator platforms.
\end{abstract}

\section{INTRODUCTION}

Teams of mobile robots that use wireless communications promise to support many new applications in the future. One possibility is a rescue situation where a team of robots must search a building for trapped humans. The search robot may be teleoperated by a human at a remote command center. The teleoperated robot must maintain a multi-hop connection through the network in order to send real-time video and receive control signals. This requires that the team automatically adjust its position to maintain a network topology suitable for the teleoperation. In addition, the connection has certain quality of service (QoS) requirements that could include: minimum bandwidth, duration of connection, and maximum allowable jitter, among others. Once a route for the QoS flow has been established, we can guarantee connectivity along the route by controlling the motion of the robots that are routing. At the same time, the robots should be able to address other tasks concurrently. We present a distributed control architecture that allows the robots to address secondary objectives while maintaining routing connectivity.

Unlike most common mobility models used in ad hoc network research [3], in this setting we assume that the nodes are able to actively move in service to networkrelated goals. The nodes can reconfigure themselves to achieve a configuration that is better-suited for the current network task, which, in this case, is maintaining QoS requirements. From the networking perspective, controllable mobility makes the ad hoc networking problem simpler, since the system is able to actively reduce the chance of a routing fault, as opposed to other mobility models, where faults are a common occurrence. In fact, it is the presence of network routing faults caused by mobility that makes ad hoc routing difficult. However, from the robotics perspective, the distributed control of a large system that must address multiple goals concurrently is a very challenging problem.

We propose a multi-objective control framework for handling QoS routing requirements in multi-robot teams. The framework addresses multiple objectives by mapping subordinate actions into the null space of superior objectives. Maintaining network QoS is the highest priority task, because for most scenarios, if the team becomes disconnected, there is no guarantee it will be able to reconnect.

However, even with controllable mobility, routing faults may still occur. For example, a robot taking part in a flow may not be able to continue routing if it needs to move outside the transmission range of its neighbors in order to address a higher priority task. Or its battery level may be too low to continue transmitting at the specified bit rate. The benefit of being able to control mobility of the nodes and be aware of their internal state is that a routing fault can be predicted before any connection is broken. Therefore, the team can actively plan to reroute traffic so that there is no loss of service.

We propose the QoS Hand Off Protocol (QHOP) for handling route discovery and repair in controllable networks. This protocol takes advantage of mobility to recruit robots that may not be located within transmission range of the host causing the fault. 


\section{RELATED WORK}

\section{A. Multi-Robot, Multi-Objective Controllers}

In previous work, we developed an architecture for multi-process control, based on the control basis [14], that allows behaviors to be constructed by combining closed-loop controllers via null space projections [17], [19]. Like the subsumption architecture [1], behavior is constructed by combining lower-level controllers. In the case of subsumption this combination is performed via inhibition and suppression. However, this approach requires the system programmer to take into account all possible control interactions at the level of their interconnection.

Burridge et al. [2] describe a scheme for robot control based on the sequential composition of Lyapunov stable controllers. Each controller works to bring the robot to a state that is within the domain of the next controller. Because of the Lyapunov stability of the constituent controllers, the robot is guaranteed to reach the goal state given that it starts in a stabilizable state. In our work, we have not solved for guaranteed domains a priori, and we require concurrent composition of controllers. In addition, our controllers are "best-effort" in the sense that the closed-loop response rejects perturbations to the system, and no destructive control interaction will destabilize the system.

The filter cascade method [20] handles multiple objectives by creating sets of filters that act to progressively reduce the input space, which is then ranked by an objective function to determine the next control action. Although useful when control actions are represented by discrete sets, in this work we use controllers represented by continuous artificial potential fields. Pirjanian [12] presents a method for multi-objective control where the control actions are determined by optimizing a weighted, linear objective function. However, linear combinations are susceptible to local minima, and there is no guarantee destructive control interactions can be avoided. Dias [6] describes a novel market-based method for multi-robot control, where robots assign values to tasks and "trade" them with other robots based on their ability to complete the tasks. Market-based methods perform well for resource allocation, which could be incorporated into the routing repair protocol described below; however, for multi-objective control, such an approach lacks the runtime property maintenance guarantees given by the control basis and required by this application.

\section{B. QoS in Mobile Ad Hoc Networks}

Routing with QoS in ad hoc networks is an active research area in the networking community [4], [9], [5]. In this paper we assume that such a capability is available to the robots, and in our simulation we use a form of Ad Hoc On-Demand Distance Vector (AODV) routing [10] that has been modified to support bandwidth-aware QoS routes [11]. However it is generally assumed that the nodes in the network have no control of their mobility with respect to routing needs. In our approach, we wish to take advantage of local state information in order to make routing decisions before failures occur. For example, if signal strength decreases, it may be due to temporary signal fluctuations, or because the sender and receiver are moving apart. Because we can examine the desired motion of the robots, we can determine whether the signal attenuation is expected to continue, and initiate route discovery if necessary.

\section{Distributed Multi-Robot CONTRol}

Let $R$ be a team of $n$ mobile robots with heterogeneous sensing capabilities and similar wireless network equipment. In this system, robots address coordinated tasks for which QoS is a critical requirement. Secondary objectives are also addressed while participating in routing a QoS flow. Some robots may be involved in routing more than one QoS flow simultaneously. Both tasks generate motor commands for the robot to move so that it stays connected to each routing neighbor. It is necessary to coordinate those actions so that they do not destructively interfere with each other.

Quality of service is a guarantee that a specified level of service will be maintained on a given connection. There are a number of different aspects of a connection that can be specified: bandwidth, connectivity time, and jitter, among others. In this paper, we examine the bandwidth and connectivity quality of service parameters. Control solutions ensure connectivity and provide the appropriate bandwidth between routing hosts. Hereafter, a "QoS flow" refers to a connection between nodes that has a specified minimum level of bandwidth and routing connectivity from source to destination.

\section{A. Control Basis Approach}

Controllers used in this work are constructed using the control basis approach [14]: a controller $\phi_{\mathcal{E}}^{\mathcal{S}}$ is constructed by associating a state estimator, $\mathcal{S}$, and effectors, $\mathcal{E}$, with an objective function, or artificial potential, $\phi$. For example, a search controller can be written:

$$
\phi_{i}^{S}
$$

where robot $i$ achieves search goal states $S$ by greedy descent on $\phi$. Assume that the robot running the search controller is also the robot that initiates a QoS flow to relay sensor data related to the search. 
The other control task examined in this paper is QoS preservation. Given a pair of robots, $i, j \in R$, and an existing QoS flow $f$, that specifies a certain minimum bit rate, $b$, between $i$ and $j$, then the goal set for a "QoS controller" should be the area where $i$ and $j$ can communicate at that bandwidth. Due to the complex, stochastic nature of RF reception, it is infeasible to compute an exact map of the QoS region between the robots. Instead, the controller uses an approximate region that can be computed in real-time, and maximizes the likelihood of high bandwidth communication.

\section{B. RF Channel Model}

To motivate the calculation of the QoS goal area, we give a brief description of the model of the transmitters and receivers in our ad hoc network. The RF signal captured at a receiver is made up of a superposition of transmitted signal and its reflections off other objects. Depending on the relative phase of these multipath waves, the interference can be constructive or destructive. The path loss is a measure of the signal attenuation from the transmitter to the receiver. Large-scale path loss is used to describe the loss in signal due to propagation over large distances and can be modeled as

$$
P_{r}=\frac{k P_{t}}{d^{n}},
$$

where $P_{r}$ and $P_{t}$ are the signal powers at the receiver and transmitter, respectively, $k$ is a constant related to the antenna gain, $d$ is the distance between the transmitter and receiver, and $n$ is typically between 2 and 4 [15]. Small-scale path loss, or fading, is a rapid fluctuation in signal quality over a short period of time, caused by reflections off objects, Doppler effects, and the relative movement of the receiver, transmitter, and objects in the environment. The effect of fading on the signal is typically modeled using a Ricean distribution,

$$
p\left(P_{r}\right)=\frac{P_{r}}{\sigma^{2}} e^{-\left(\frac{P_{r}^{2}}{2 \sigma^{2}}+K\right)} I_{0}\left(2 K P_{r}\right),
$$

where $K$ represents the peak amplitude of the dominant signal and $I_{0}(\cdot)$ is the modified Bessel function of the first kind and zero-order [15]. The $K$ parameter denotes the effect of the line of sight (LOS) portion of the signal. The total path loss is calculated as the sum of the large and small scale path loss.

Thus we see that both line of sight and distance between transmitter and receiver affect the received signal strength. We construct our controllers such that the large scale path loss is minimized by the QoS controller, and line of sight is maintained to minimize the fading of the signal.

\begin{tabular}{|c|c|c|c|}
\hline 11 Mbps & 5.5 Mbps & 2 Mbps & 1 Mbps \\
\hline $25 \mathrm{~m}$ & $35 \mathrm{~m}$ & $40 \mathrm{~m}$ & $50 \mathrm{~m}$ \\
\hline
\end{tabular}

TABLE I

NOMINAL TRANSMISSION RATES AS A FUNCTION OF DISTANCE, FOR THE ORINOCO TRANSCEIVER, IN AN ENCLOSED ENVIRONMENT.

We assume that robots in $R$ are equipped with $802.11 \mathrm{~b}$ transceivers. The $802.11 \mathrm{~b}$ specification allows for multirate transmission, at $11,5.5,2$, or 1 Mbps based on the received signal strength. The standard does not specify an algorithm for selecting the current transmission rate [8]. Although the details of how the transmission rate is calculated is beyond the scope of this paper, given the above model, the signal strength is related to the distance and line of sight properties between transmitter and receiver. Furthermore, we assume that the greater the received signal strength, the higher the allowable bit rate. There is research into multi-rate adaption algorithms [16], but for our purposes we adopt the specifications given by the manufacturer of our transceiver, the Orinoco brand PC Card adapter [13]. Table I shows the data rates as a function of receiver distance in an enclosed area, taken from [13].

\section{QoS Controller}

Given the above channel model, the possible bandwidth between a pair of robots is a function of the distance between the pair and whether they are within LOS. Let the transmission range on robot $i$ for bandwidth level $b$ be denoted $r_{b}^{i}$. The QoS goal set $\mathcal{Q}_{i j}^{f}$ is computed as

$$
\mathcal{Q}_{i j}^{f}=L O S_{i j} \wedge Q_{i j}^{f},
$$

where $L O S_{i j}$ is the LOS region between $i$ and $j$, and $Q_{i j}^{f}$ is a circle with radius $\min \left\{r_{b}^{i}, r_{b}^{j}\right\}$, which is the region where $i$ and $j$ can communicate with bandwidth $b$. Both $L O S_{i j}$ and $Q_{i j}^{f}$ are centered about robot $i$. For $j$ to compute $\mathcal{Q}_{i j}^{f}, i$ must send the pair $\left\langle L O S_{i j}, r_{b}^{i}\right\rangle$.

The shape of $L O S_{i j}$ depends on the sensing abilities of robot $i$. For example, if $i$ has only local proximity detection sensors, then $i$ may only be able to sense freespace within $1 \mathrm{~m}$ of the robot, which may be a small subset of the QoS area. If robot $i$ uses a high-resolution camera or laser range finder for determining the LOS region, then it may be able to specify a large LOS region that subsumes multiple levels of QoS bandwidth. If a map of the environment is known, then a robot can calculate the true LOS region between it and its peer, which may be greater than the range of its sensors. This 
allows a longer range, with less bandwidth used between robots to communicate the QoS area.

Given the QoS goal set $\mathcal{Q}_{i j}^{f}$, the QoS controller is

$$
\phi_{j}^{\mathcal{Q}_{i j}^{f}},
$$

where robot $j$ must achieve QoS-maintaining states $\mathcal{Q}_{i j}^{f}$ by greedy action on $\phi$.

\section{Coordinated, Distributed Controllers}

So far we have described two controllers for accomplishing two distinct tasks, searching and QoS maintenance. Robots involved in routing a flow may need to perform multiple tasks concurrently. A method is needed for eliminating destructive control interactions; in other words, allowing both controllers to run concurrently while ensuring they both continue to make progress toward their goals.

A team of mobile robots can be viewed in a control framework as a redundant system with many degrees of freedom. We use the control framework presented in [17], [19]. The system Jacobian for the team may be redundant, which allows secondary tasks to be addressed by projecting secondary control actions onto the null space of the Jacobian. This requires that all addressable tasks be arranged in increasing order of priority, so that subordinate tasks may be projected onto the null space of superordinate tasks. Each robot determines its prioritization of tasks independently, which may be based on local state information. For example, a robot may prioritize tasks based on the expected probability of success, which it calculates from local state such as its battery level.

Since control actions are derived by descending artificial potentials, a secondary control action will not interfere if it moves the robot along an equipotential line of the primary control potential. This is similar to projecting secondary control actions onto the null space of the primary controller, so that the new action does not interfere with the primary control action. The "subjectto" operator, $\triangleleft$, performs the null space projection. For example, a robot $i$ that is maintaining a QoS flow with robot $j$ may also wish to perform a search concurrently. In this case, QoS-maintenance is the primary task, so all search actions are projected onto the null space of the QoS controller. The combined, pairwise controller is written as

$$
\phi_{i}^{S} \triangleleft \phi_{j}^{\mathcal{Q}_{i j}^{f}} .
$$

This controller will allow $i$ to search while $j$ maintains QoS by moving to $\mathcal{Q}_{i j}^{f}$. The robots move as a pair where robot $i$ is the leader, since it is specifying $\mathcal{Q}_{i j}^{f}$ to $j$. While "following" $i$, robot $j$ may address secondary objectives using the null space projection.

In addition to two robots, multiple robots can form a serial, kinematically-related chain by combining QoS controllers. Let robots $i, j$, and $k$ be a chain which routes a flow from $i$ to $k$. Robot $i$ is the source of the flow and executes the controller in (6), while the middle robot $j$ is involved in a pairwise "pull" controller [17] with both neighbors:

$$
\phi_{j}^{\mathcal{Q}_{i j}^{f}} \triangleleft \phi_{k}^{\mathcal{Q}_{j k}^{f}} .
$$

The combination of controllers (6) and (7) allows the three-robot chain to move anywhere about the environment while maintaining QoS connectivity.

In some circumstances, we would like the movement of the leader to be limited to an area specified by a follower robot; for instance, if the base of the chain is an immobile hub, then its leader should not be allowed to move beyond its communication range. If we designated robot $k$ as the hub, then the "push" controller [17] with robot $j$ could be written as

$$
\phi_{j}^{\mathcal{Q}_{i j}^{f}} \triangleleft \phi_{j}^{\mathcal{Q}_{j k}^{f}} .
$$

If the team were to execute controllers (6) and (8), then the exploration would be constrained by the movement of the hub, $k$.

For an arbitrary QoS flow $f$ through $R$, each robot along the route of $f$ must execute a QoS controller with its up- and down-stream neighbors. The chain constrains the movements of its members to within those areas that maintain QoS requirements for $f$. The maximum number of robots that can form such a QoS chain depends on the real-time process requirements of the robots. This bound is discussed in more detail in [19].

\section{E. QoS Threshold}

Assuming robots $i$ and $j$ are executing the controller in (6), if $j$ is not within the QoS region $\mathcal{Q}_{i j}^{f}$, then a routing fault will occur if the specified bandwidth for $f$ cannot be achieved between the robots. Consequently, $j$ may predict when a routing fault will occur as it approaches the boundary of $\mathcal{Q}_{i j}^{f}$. If $j$ cannot continue routing $f$, we would like to able to recruit some other member of $R$ to take its place in routing the flow. For this to occur, $j$ must act to recruit a replacement before the fault occurs. We define the QoS threshold region $R F_{i j}^{f} \subseteq \mathcal{Q}_{i j}^{f}$ that will serve as an enlarged routing-fault boundary. Upon entering $R F_{i j}^{f}$, robot $j$ should initiate the QoS routing hand off protocol described in Section IV. The size of $R F_{i j}^{f}$ should vary depending on the speed of $j$; $R F_{i j}^{f}$ should increase as $j$ moves faster, 


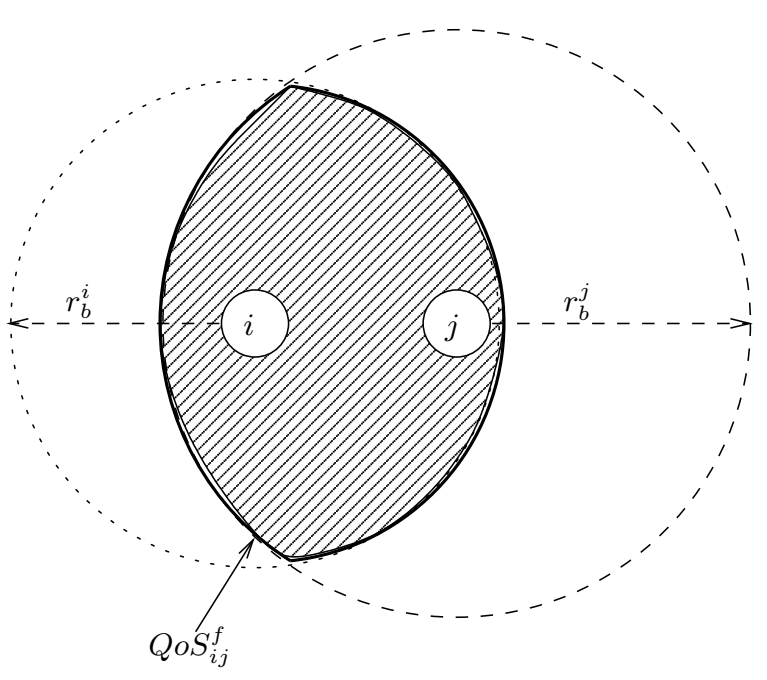

Fig. 1. The QoS region $\mathcal{Q}_{i j}^{f}$ for two robots $i$ and $j$ using a transmission rate of $r_{b}$. The two transmission radii are unequal, representing differing transmission power.

since more time is required to perform a routing hand off.

It may be unavoidable for a robot to move into the $R F$ area; for example, if it must avoid a dynamic obstacle. The robot can decide, based on its desired movement vector, whether it will remain in the $R F$ area, or it returns to the QoS goal area. By having this local state, unnecessary rerouting can be avoided.

\section{QOS FLOW HAND OFF}

The robot team has a certain network topology based on connectivity and QoS capabilities. We are interested in determining how the team may reconfigure itself to address new tasks. For example, given a QoS flow $f$, some participant $j$ may not be able to continue servicing $f$, in which case another robot could be recruited to take over for $j$. Situations that could cause such a failure include a robot's battery level falling below the level at which it needs in order to transmit at the given QoS, or it may have another, more important, objective to address that keeps it from moving into the desired region, $\mathcal{Q}_{i j}^{f}$, necessary to continue routing.

It is desirable if another robot with available resources can join in the existing flow to take the place of the failing member. A protocol is needed to determine which team member will take over, and how the hand off should occur so as to make sure that QoS of the flow is maintained. We have developed the QoS Hand Off Protocol (QHOP) to implement this functionality. The general idea is that the robot that will be leaving the flow broadcasts a request for another robot to take its place.
This requires that some other robot within the team be available to move to the location of the requesting robot. Once the robot has arrived, a hand off procedure is initiated that performs a local rerouting around the exiting robot while ensuring packets are not lost during the hand off. An in-depth description of the protocol is beyond the scope of this paper, but more information can be found in [18].

\section{EVAluation}

\section{A. Simulator Platform}

In order to simulate a robotic, mobile, ad hoc network, we must simulate the appropriate network conditions as well as the control of the robots. To achieve this, we merged the simulation capabilities of a network simulator, ns-2, and a mobile robot simulator, Player/Stage [7]. The Player/Stage simulator simulates the mobility and sensing aspects of the robot team, while ns-2 simulates the ad hoc network traffic and QoS capabilities of the robots. In this simulator, only large scale path loss (equation (2)) is modeled; small scale fading effects on the RF signal are ignored.

We have implemented the QoS controllers in Player/Stage, and the QHOP protocol for $802.11 \mathrm{~b}$ wireless nodes in ns-2. The simulators are coordinated via shared memory so that at each simulated time step, ns-2 nodes receive position information from their counterpart robots in Player/Stage. Using this position information, ns-2 simulates the routing performance and current bandwidth level, which is passed back to the Player/Stage nodes. The Player/Stage simulated robots adjust their position based on the network information from ns-2.

This work assumes that the robots have some sort of QoS ad hoc routing capabilities in place. For our evaluation we developed a QoS ad hoc routing protocol based on Ad Hoc On Demand Distance Vector (AODV) routing, described in [10].

\section{B. QoS Control with Hand Off}

To demonstrate the QoS controllers and QHOP protocol, we performed a simulation with four robots in a $50 \mathrm{~m} \times 50 \mathrm{~m}$ environment. Each robot is a differential drive mobile robot equipped with a laser range finder. The leader, a teleoperated search robot, initiates a flow to the hub requiring $11 \mathrm{Mbps}$ that results in a serial routing configuration between all four robots. The robots must try to maintain QoS and LOS constraints while moving in the environment. The fourth robot in the chain acts as a stationary hub. The leader, Robot 0 , and the middle robot, Robot 1 , use a pull relationship that allows the leader to search the environment, shown in 
equation (9). Robots 1 and 2 use a pull controller, shown in equation (10), while Robot 2 and the hub use a push controller, shown in equation (11):

$$
\begin{aligned}
\phi_{0}^{S} & \triangleleft \phi_{1}^{\mathcal{Q}_{01}^{f}}, \\
\phi_{1}^{\mathcal{Q}_{01}^{f}} & \triangleleft \phi_{2}^{\mathcal{Q}_{12}^{f}}, \\
\phi_{2}^{\mathcal{Q}_{12}^{f}} & \triangleleft \phi_{2}^{\mathcal{Q}_{23}^{f}} .
\end{aligned}
$$

The QoS region was set according to Table I, which for $11 \mathrm{Mbps}$ in an enclosed area is $25 \mathrm{~m}$. Each laser range finder has a maximum range of $80 \mathrm{~m}$, however the LOS region was set to $25 \mathrm{~m}$, so that if the robots are within LOS then they should be close enough to transmit data at 11 Mbps. Figure 2 shows the trajectories of the team within the environment. The search controller on Robot 0 was given two waypoints, to simulate the behavior of a teleoperator; the first is in the upper right quadrant, and the second in the upper left. Robot 0 must use Robot 1 as an intermediate communications node if it wishes to transmit to the hub. Figures 3-5 show the received signal strength (RSS) between sender and receiver pairs of robots during the task. The solid line indicates the RSS of data packets, while the dashed line represents the minimum RSS necessary for $11 \mathrm{Mbps}$ transmission. The minimum was computed as the signal strength at $25 \mathrm{~m}$ given a transmission power of $15 \mathrm{dBm}$; this is more conservative than the actual wireless card, which claims a sensitivity of $-82 \mathrm{dBm}[13]$. The graphs show that the robots are able to maintain the $11 \mathrm{Mbps}$ level throughout the task.

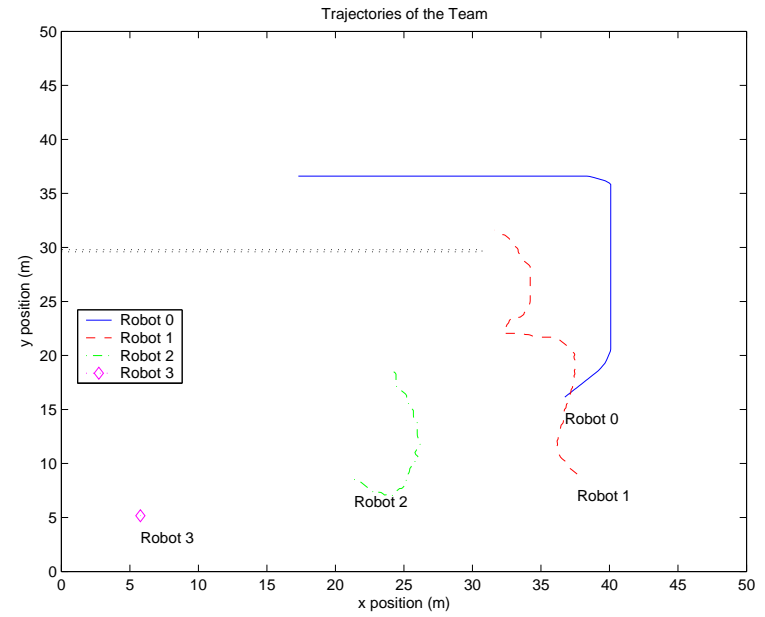

Fig. 2. This shows the trajectories of the team of four robots during the task. The label beneath the trajectory indicates the starting position of the robot. The world is $50 \mathrm{~m} \times 50 \mathrm{~m}$, with an obstacle indicated by the horizontal line.

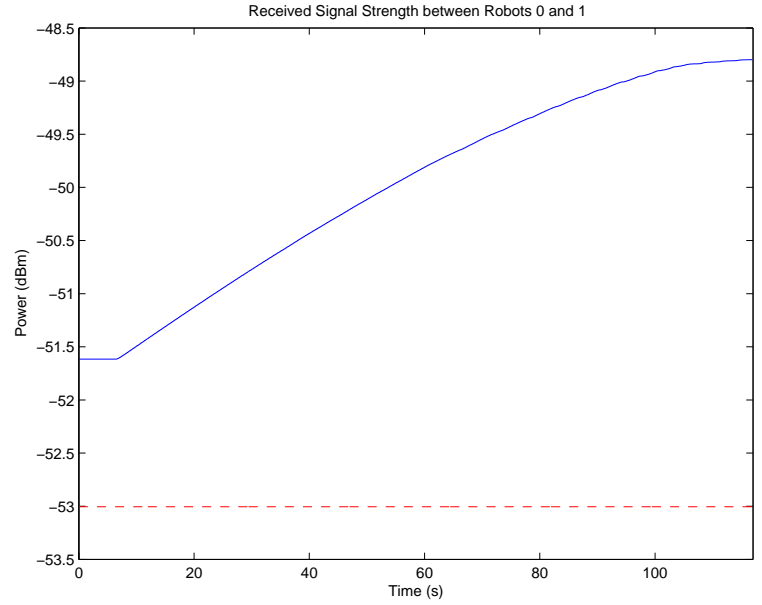

Fig. 3. This graph shows the received signal strength (RSS), shown as a solid line, measured in $\mathrm{dBm}$, between robots 0 and 1 . This level is compared to the minimum RSS required for $11 \mathrm{Mbps}$ transmission (dashed line).

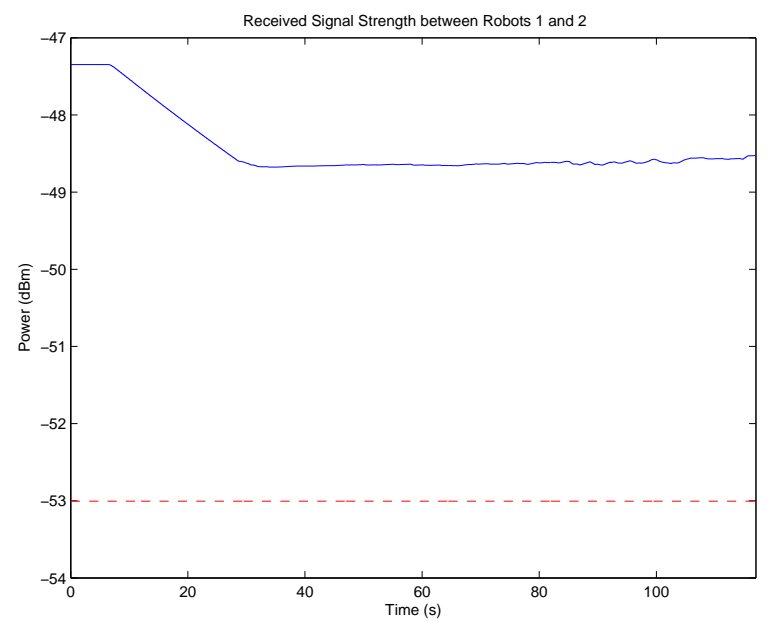

Fig. 4. This graph shows the RSS between robots 1 and 2 (solid line), compared to the minimum required RSS (dashed line) for $11 \mathrm{Mbps}$ transmission.

\section{QoS Routing Hand Off}

We developed another simulated scenario to demonstrate QoS routing hand off using QHOP. In this experiment, four robots are used, with three of the robots implementing the pull controller. After some time, one of the robots detects a likely routing fault and initiates a hand off request. The fourth robot responds to the request and joins the chain. Figure 6 shows the paths of the four robots in the environment. The arrow indicates the starting position of Robot 3, the robot that initiates the QHOP hand off. In this example, the start of the 


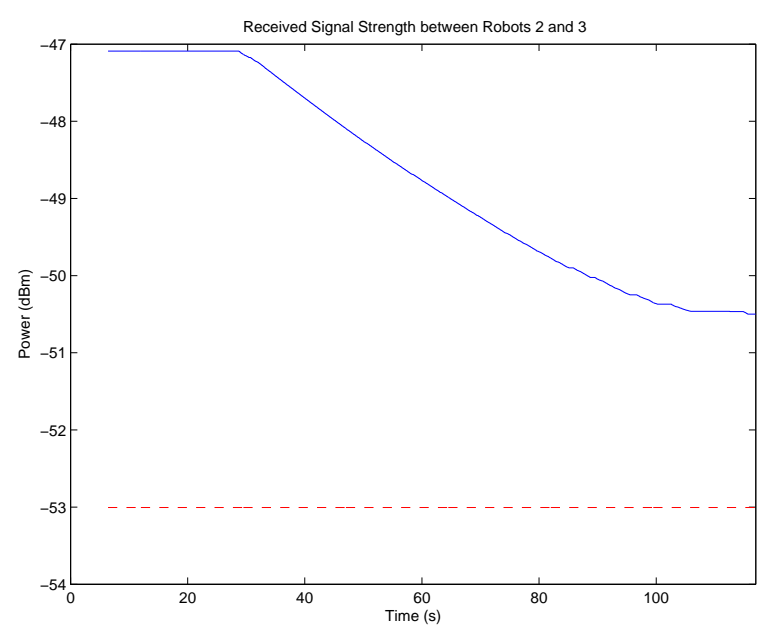

Fig. 5. This graph shows the RSS between robot 2 and 3 (solid line), compared to the minimum required RSS (dashed line) for $11 \mathrm{Mbps}$ transmission.

hand off process was chosen arbitrarily. Figure 7 shows the differences in distance between the four robots as they execute the task. The maximum QoS and LOS distance is marked with a dashed line. The discontinuity represents the position where the robot that performs the routing hand off, Robot 3, joins the LOS chain and starts to follow Robot 0 . In this example, there were no boundary regions for the LOS and QoS goals, which results in the two follower robots periodically exceeding the QoS and LOS boundary. This also illustrates the best-effort nature of the controller; as soon as the goal region is lost, the robot acts to return to the goal region.

\section{CONCLUSION}

We have presented a set of distributed controllers that maintain QoS commitment between pairs of mobile robots involved in an ad hoc network. The controllers handle multiple objectives by combining control actions through a generalized null space projection. We have demonstrated the controllers in simulation.

We have also presented the QHOP link layer protocol for proactive routing path in response to possible routing faults in mobile ad hoc networks. This protocol acts to find new nodes to continue routing when a member of a QoS-constrained flow can not maintain its QoS commitments. We have demonstrated the use of the protocol in the context of QoS routing with mobile robots.

\section{ACKNOWLEDGMENT}

This work has been funded in part by DARPA projects DABT63-99-1-0022 and DABT63-00-1-0004, and by

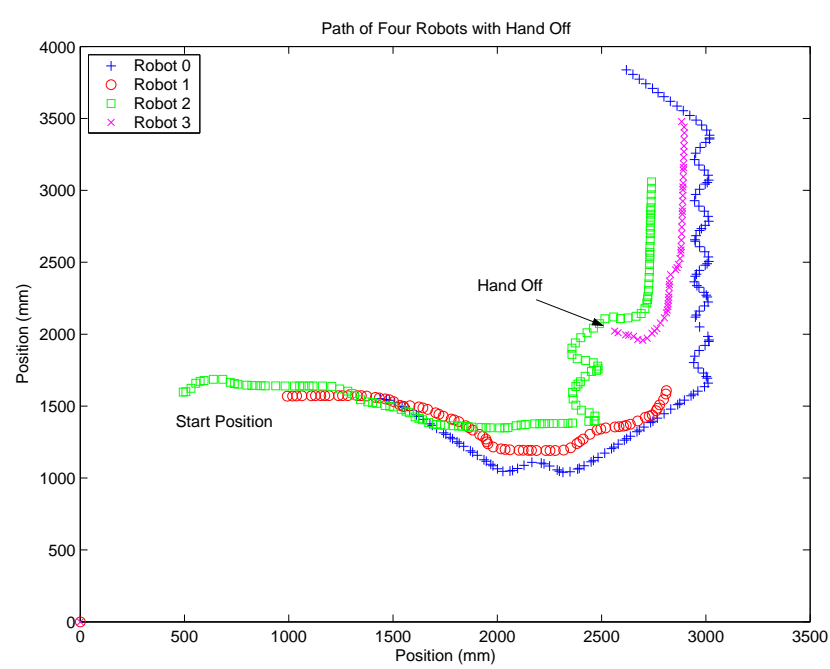

Fig. 6. This graph shows the paths of the four robots. They start in a linear configuration near the middle of the workspace. The arrow indicates the location of the robot that performs the routing hand off.

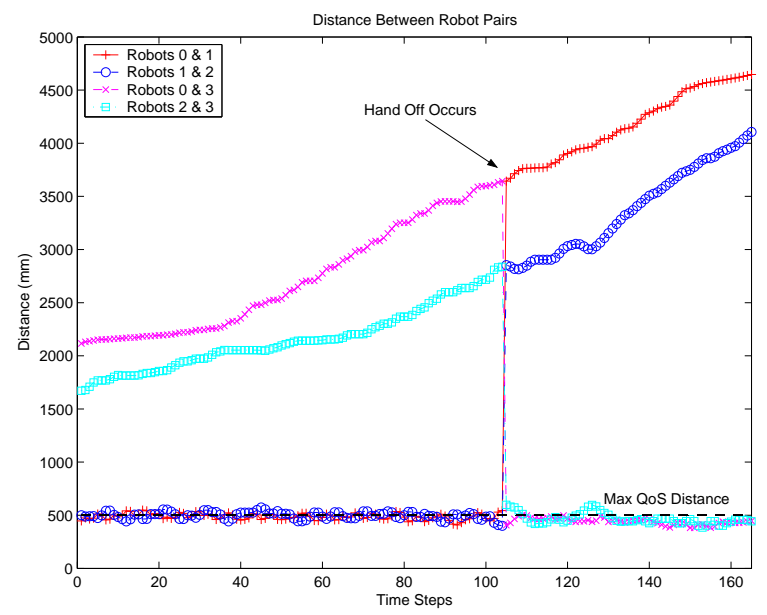

Fig. 7. This graph shows the differences in distance between the four robots involved in the task over time. The dashed line shows the maximum QoS distance. The discontinuity shows the time at which the hand off occurs, and the new robot joins the LOS chain.

the National Aeronautics and Space Administration, sponsor reference number NAG9-1445.

\section{REFERENCES}

[1] R. Brooks. A robust layered control system for a mobile robot. IEEE Journal of Robotics and Automation, 2(1):14-23, March 1986.

[2] R. R. Burridge, A. A. Rizzi, and D. E. Koditschek. Sequential composition of dynamically dexterous robot behaviors. The International Journal of Robotics Research, 18(6):534-555, June 1999. 
[3] T. Camp, V. Davies, and J. Boleng. A survey of mobility models for ad hoc network research. Wireless Communication \& Mobile Computing (WCMC): Special issue on Mobile Ad Hoc Networking: Research, Trends and Applications, 2(5):483-502, 2002.

[4] S. Chen and K. Nahrstedt. Distributed quality-of-service routing in ad-hoc networks. IEEE Journal on Selected Areas in Communications, 17(8), August 1999. Special Issue on Ad-Hoc Networks.

[5] T.-W. Chen, J. Tsai, and M. Gerla. QoS routing performance in multihop, multimedia, wireless networks. In Proceedings of ICUPC '97, 1997.

[6] M. B. Dias and A. Stentz. Opportunistic optimization for marketbased multirobot control. In Proceedings of the IEEE/RSJ International Conference on Intelligent Robots and Systems (IROS). IEEE, 2002.

[7] B. Gerkey, R. T. Vaughn, and A. Howard. The player/stage project: Tools for multi-robot and distributed sensor systems. In Proceedings of the 11th International Conference on Advanced Robotics, June 2003.

[8] IEEE. Part 11: Wireless LAN Medium Access Control (MAC) and Physical Layer (PHY) specifications: Higher-Speed Physical Layer Extension in the 2.4 Ghz Band, 1999. IEEE Std 802.11b1999.

[9] S.-B. Lee, G.-S. Ahn, X. Zhang, and A. T. Campbell. INSIGNIA: An IP-based quality of service framework for mobile ad hoc networks. Journal of Parallel and Distributed Computing, 60:374-406, 2000.

[10] C. Perkins and E. M. Royer. Ad-hoc on-demand distance vector routing. In MILCOM '97 panel on Ad Hoc Networks, November 1997.

[11] C. E. Perkins, E. M. Royer, and S. R. Das. Quality of Service for Ad Hoc On-Demand Distance Vector Routing. IETF Mobile Ad Hoc Networking Group, July 2000. draft-ietf-manet-aodvqos00.txt.

[12] P. Pirjanian and H. I. Christensen. Behavior coordination using multiple-objective decision making. Sensor Fusion and Decentralized Control in Autonomous Robotic Systems, 3209:78-89, October 1997.

[13] Proxim Corporation. ORiNOCO 11b Client PC Card, 2003.

[14] G. RA, H. M, J. J. Coelho, and S. K. Distributed control of manipulation tasks. IEEE Expert, Special Track on Intelligent Robotic Systems, 10(2):9-14, 1995.

[15] T. S. Rappaport. Wireless Communications: Principles and Practice. Prentice-Hall PTR, second edition edition, 2002.

[16] B. Sadeghi, V. Kanodia, A. Sabharwal, and E. Knightly. Opportunistic media access for multirate ad hoc networks. In Proceedings of MOBICOM '02. ACM, September 2002.

[17] J. Sweeney, T. Brunette, Y. Yang, and R. A. Grupen. Coordinated teams of reactive mobile platforms. In Proceedings of the IEEE International Conference on Robotics and Automation (ICRA). IEEE, May 2002.

[18] J. D. Sweeney, R. A. Grupen, and P. Shenoy. Active QoS flow maintenance in robotic, mobile, ad hoc networks. Technical Report 04-20, University of Massachusetts Amherst, April 2004.

[19] J. D. Sweeney, H. Li, R. A. Grupen, and K. Ramamritham. Scalability and schedulability in large, coordinated, distributed robot systems. In Proceedings of the IEEE International Conference on Robotics and Automation (ICRA). IEEE, September 2003.

[20] B. J. Thibodeau, S. W. Hart, D. R. Karuppiah, J. D. Sweeney, and O. Brock. Cascaded filter approach to multi-objective control. In Proceedings of the IEEE International Conference on Robotics and Automation (ICRA). IEEE, April 2004. 